\title{
Velocity Slip and Temperature Jump in Hypersonic Aerothermodynamics
}

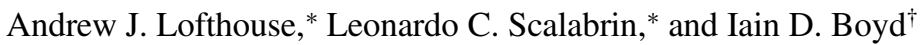 \\ University of Michigan, Ann Arbor, Michigan 48109
}

DOI: $\underline{10.2514 / 1.31280}$

\begin{abstract}
Hypersonic vehicles experience different flow regimes during flight due to changes in atmospheric density. Computational fluid dynamics, although relatively computationally inexpensive, is not physically accurate in areas of highly nonequilibrium flows. The direct simulation Monte Carlo method, although physically accurate for all flow regimes, is relatively computationally expensive. In a continuing effort to understand the performance of computational fluid dynamics and direct simulation Monte Carlo in hypersonic flows, the current study investigates the effect of continuum breakdown on surface aerothermodynamic properties (pressure, shear stress, and heat transfer rate) of a cylinder in Mach-10 and Mach-25 flows of argon gas for several different flow regimes, from the continuum to a rarefied gas. Several different velocity-slip and temperature-jump boundary conditions are examined for use with the computational fluid dynamics method. Computational fluid dynamics and direct simulation Monte Carlo solutions are obtained at each condition. Total drag and peak heat transfer rate predictions by computational fluid dynamics remain within about $6 \%$ of the direct simulation Monte Carlo predictions for all regimes considered, with the Gökçen-type slip condition giving the best results.
\end{abstract}

\section{Introduction}

$\mathbf{T}$ HE design of hypersonic vehicles requires accurate prediction of the surface properties. These quantities are typically the heat flux, pressure, and shear stress. During its trajectory through an atmosphere, a hypersonic vehicle will experience vastly different flow regimes due to the variation of atmospheric density with altitude. Reproduction of these varied flow conditions in groundbased laboratory facilities is both expensive and technically challenging. Hence, computational modeling of these flows has become extremely important in the development of hypersonic vehicles.

At low altitudes, the atmospheric density is relatively high, and flows around hypersonic vehicles should be simulated using traditional computational fluid dynamics (CFD) by solving the Navier-Stokes (NS) equations. This is the continuum regime characterized by very large Reynolds numbers and very low Knudsen numbers. The Knudsen number is defined as the ratio of the mean free path to a characteristic length, $K n=\lambda / L$, where the mean free path $\lambda$ is inversely proportional to the density. At very high altitudes the density is low, such that there are very few collisions between the molecules and atoms in the flow around the vehicle. This is the rarefied flow regime, characterized by larger Knudsen numbers, and can be computed using the direct simulation Monte Carlo (DSMC) method [1]. Generally speaking, CFD methods for solving the NS equations are about an order of magnitude faster than the DSMC method. However, the lack of collisions makes the physics of the NS equations invalid in rarefied regimes. Additionally, on a blunt body, a high-density forebody flow can create a rarefied flow in the wake of the vehicle. In principle, the DSMC method can be applied to any dilute gas flow, but becomes prohibitively expensive for Knudsen numbers less than about 0.001 .

Presented as Paper 208 at the 45th AIAA Aerospace Sciences Meeting and Exhibit, Reno, NV, 8-11 January 2007; received 28 March 2007; revision received 12 July 2007; accepted for publication 12 July 2007. This material is declared a work of the U.S. Government and is not subject to copyright protection in the United States. Copies of this paper may be made for personal or internal use, on condition that the copier pay the $\$ 10.00$ per-copy fee to the Copyright Clearance Center, Inc., 222 Rosewood Drive, Danvers, MA 01923; include the code 0887-8722/08 \$10.00 in correspondence with the CCC.

*Graduate Student, Department of Aerospace Engineering. Senior Member AIAA.

Graduate Student, Department of Aerospace Engineering. Student Member AIAA.
Thus, it is attractive to find ways to increase the validity of CFD methods beyond the continuum regime.

CFD modeling in the transition regime (that is, for lower density flows beyond the continuum regime but before the free-molecular regime) can be improved by replacing the typical no-slip boundary conditions at a surface with velocity-slip and temperature-jump boundary conditions. These boundary conditions will not, however, eliminate all sources of errors when using continuum methods for flows far from equilibrium. For instance, in more rarefied flows, the pressure tensor ceases to be isotropic, which is a key assumption in the derivation of the continuum equations.

The areas of the flow in which the continuum hypothesis breaks down (or equivalently, in which the flow is no longer in local thermodynamic equilibrium) can be quantified by the use of a continuum breakdown parameter. Although there have been several breakdown parameters presented in the literature [2-5], the one that appears to be most appropriate for hypersonic compressible flows is the gradient-length local (GLL) Knudsen number [ㅁ, $]$ ]:

$$
K n_{\mathrm{GLL}}=\frac{\lambda}{Q}\left|\frac{\mathrm{d} Q}{\mathrm{~d} l}\right|
$$

where the derivative is taken in the direction of the maximum gradient, and $Q$ is some quantity of interest such as density, pressure, temperature or velocity magnitude. When calculating $K n_{\mathrm{GLL}}$ based on velocity magnitude, the gradient is normalized by the maximum of the local velocity magnitude and the local speed of sound. It is generally assumed that continuum breakdown occurs whenever $K n_{\text {GLL }}$ (based on the CFD solution) is greater than 0.05 .

Previous work [8] sought to quantify the errors in CFD predictions of a hypersonic blunt body's surface properties by comparing those solutions with DSMC solutions for a variety of flows in the transitional regime. The maximum $K n_{\mathrm{GLL}}$ was also computed in each case to determine the effect of continuum breakdown on the surface properties. The cases considered were limited to a Mach-10 flow of argon, with no-slip boundary conditions for the CFD solutions. As would be expected, the results indicated that the inclusion of velocity-slip and temperature-jump boundary conditions would improve the agreement between the DSMC and CFD conditions.

The current work builds on these results, with the main objective being to quantify the improvement in agreement between the DSMC solutions and the CFD solutions with the inclusion of slip boundary conditions. Additional cases of a Mach-25 flow are also computed to 
determine any effects of continuum breakdown at higher velocities on the surface properties.

We first briefly describe the simulation procedures used, including some background of the computational models. Several slip boundary conditions are then evaluated. We then discuss general flowfield and surface property results predicted by the models in several different flow regimes, from the continuum to a rarefied gas. Finally, some conclusions are presented.

\section{Background and Simulation Procedure}

This investigation considers a hypersonic flow of argon over a two-dimensional, 12-in.-diam cylinder, as shown in Fig. 1. Two different freestream velocities are used that correspond to Mach-10 and Mach-25 flows ( $U_{\infty}=2624$ and $6585 \mathrm{~m} / \mathrm{s}$, respectively). The wall temperature is held constant at $500 \mathrm{~K}$ (Mach 10) and $1500 \mathrm{~K}$ (Mach 25). The freestream density of the flow is varied such that several different regimes are considered, from the continuum through the transitional to the rarefied regime, as shown in Table 1 . Knudsen numbers are calculated based on freestream conditions and the cylinder diameter, using the hard-sphere model for the meanfree-path calculation. Surface and flowfield properties for this flow are presented from two different computational approaches.

First, DSMC solutions are generated using the MONACO code [9] for each case shown in Table 1 . MONACO is a general $2 \mathrm{D} / 3 \overline{\mathrm{D}}$, parallel, unstructured-mesh DSMC code that has been applied to many hypersonic, rarefied flows [10].

The variable hard-sphere (VHS) [1] parameters used in the DSMC computations are equivalent to those in the previous work: the temperature exponent $\omega$ is 0.734 , with a reference diameter of $3.595 \times 10^{-10} \mathrm{~m}$ at a reference temperature of $1000 \mathrm{~K}$. In general, the mesh used for the final solution for each case is adapted from previous solutions such that each cell size is on the order of a mean free path. The exceptions are the $K n=0.002$ cases, in which the cell sizes are approximately four times the mean free path, and the $K n=0.01$ cases, in which the cell sizes near the surface are on the order of two mean free paths. In these cases, the subcell method [1] is used to select particles for collisions to ensure physical accuracy.

Second, CFD results are obtained through solution of the NavierStokes equations. The CFD results are obtained using the Michigan aerothermodynamic Navier-Stokes (LeMANS) code, developed for the simulation of hypersonic reacting flowfields $[11,12]$. LeMANS is a general 2D/3D, parallel, unstructured finite volume CFD code capable of simulating gases in thermal and chemical nonequilibrium. Different boundary conditions, including no-slip, slip velocity, and temperature jump, are enforced. These will be discussed in more detail later. In each case, a grid-independence study was conducted to determine the final mesh resolution used.

The viscosity of pure argon in the CFD code is computed to exactly correspond to the VHS model, according to Eqs. (2) and (3) [13]:

$$
\begin{gathered}
\mu=\mu_{\text {ref }}\left(\frac{T}{T_{\text {ref }}}\right)^{\omega} \\
\mu_{\text {ref }}=\frac{15 \sqrt{\pi m k T_{\text {ref }}}}{2 \pi d_{\text {ref }}^{2}(5-2 \omega)(7-2 \omega)}
\end{gathered}
$$

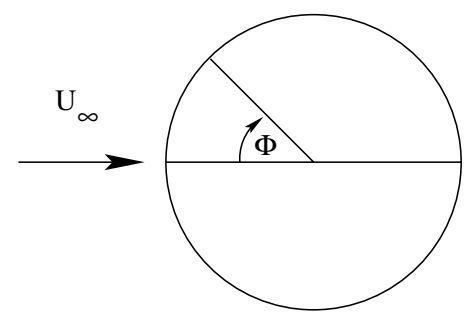

Fig. 1 Geometry definition.
Table 1 Flow regimes considered

\begin{tabular}{lcc}
\hline \hline$K n_{\infty}{ }^{\text {a }}$ & Mass density, $\mathrm{kg} / \mathrm{m}^{3}$ & $\begin{array}{c}\text { Number density, } \\
\text { particles } / \mathrm{m}^{3}\end{array}$ \\
\hline 0.002 & $1.408 \times 10^{-4}$ & $2.124 \times 10^{21}$ \\
0.01 & $2.818 \times 10^{-5}$ & $4.247 \times 10^{20}$ \\
0.05 & $5.636 \times 10^{-6}$ & $8.494 \times 10^{19}$ \\
0.25 & $1.127 \times 10^{-6}$ & $1.699 \times 10^{19}$ \\
\hline
\end{tabular}

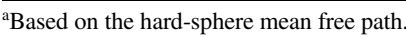

where $\mu$ is the coefficient of viscosity, $T$ is the translational temperature, $\omega$ is the VHS temperature exponent, $m$ is the mass of one molecule, $k$ is the Boltzmann constant, and $d$ is the molecular diameter. The thermal conductivity is calculated assuming a constant Prandtl number, $\mathrm{Pr}=2 / 3$.

\section{Slip Boundary Conditions}

Several different types of slip boundary conditions are implemented in the LeMANS code. The first is based on the simple slip condition first derived by Maxwell for a flat plate, as described in [14]. For an isothermal wall, this boundary condition is given as

$$
V_{s}=\left.A\left(\frac{2-\sigma}{\sigma}\right) \lambda \frac{\partial u_{x}}{\partial n}\right|_{0}
$$

where $n$ is the coordinate normal to the wall, $V_{s}$ is the slip velocity, $A$ is a constant, $\sigma$ is the momentum accommodation coefficient, and $u_{x}$ is the velocity in the $x$ direction. The mean free path $\lambda$ is calculated from typical gas flow properties, as shown in Eq. (ㅁ) [15]:

$$
\lambda=\frac{2 \mu}{\rho \bar{c}}=\frac{\mu}{\rho} \sqrt{\frac{\pi}{2 R T}}
$$

where $\bar{c}$ is the mean molecular speed and $R$ is the gas constant.

The boundary condition for temperature jump is similar [16]

$$
T_{0}-T_{w}=\left.\frac{2-\alpha}{\alpha} \frac{2 \gamma}{(\gamma+1) P r} \lambda \frac{\partial T}{\partial n}\right|_{0}
$$

where $\alpha$ is the thermal accommodation coefficient, $\gamma$ is the ratio of specific heats, and $P r$ is the Prandtl number. The thermal accommodation coefficient $\alpha$ can be assumed to be the same as the momentum accommodation coefficient $\sigma$. In this case, the second and third terms on the right-hand side can be combined and an equivalent mean free path can be adopted, as

$$
\lambda_{T}=\frac{4}{(\gamma+1)} \frac{\kappa}{\rho \bar{c} C_{v}}=\frac{2}{(\gamma+1)} \frac{\kappa}{\rho C_{v}} \sqrt{\frac{\pi}{2 R T}}
$$

where $\kappa$ is the thermal conductivity, and $C_{v}$ is the specific heat at constant volume, to give the simpler form shown in Eq. (8):

$$
T_{0}-T_{w}=\left.\frac{2-\sigma}{\sigma} \lambda_{T} \frac{\partial T}{\partial n}\right|_{0}
$$

The simple Maxwell slip conditions given by Eqs. (4) and (8) are implemented in LeMANS and referenced later as the type-1 slip condition.

The simple slip boundary conditions were originally derived by assuming a small local Knudsen number. Gökçen and MacCormack [15] showed that for large local Knudsen numbers, surface properties computed using these simple slip boundary conditions converge to different values from those predicted by free-molecular flow. They proposed the general slip boundary conditions given in Eq. (9):

$$
\sigma\left(a_{\lambda}-a_{w}\right)=\left.2 \lambda_{a} \frac{\partial a}{\partial n}\right|_{0}
$$

where $a$ is either velocity or temperature, and $\lambda_{a}$ is given by either Eq. (5) or Eq. (7). For small local Knudsen numbers, this expression 
reduces to Eqs. (4) and (ㅁ) [16]. This slip condition is referenced later in the results as the type-2 slip condition.

The Navier-Stokes equations fail in at least two different areas as the flow becomes more rarefied. The first area, an assumption of noslip flow, is corrected through the use of the slip boundary conditions. However, the NS equations also assume that the shear stress varies linearly with the velocity gradient at the wall.

Lockerby et al. [17] proposed a wall-function type of boundary condition intended to correct this second source of error. Here, the viscosity in the Knudsen layer is modified as $\mu=\mu \Psi^{-1}$, where $\Psi$, given in Eq. (10), is derived from a curve fit to the Knudsen-layer velocity profile originally derived by Cercignani, as described in [17].

$$
\Psi\left(\frac{n}{\lambda}\right) \approx 1+\frac{7}{10}\left(1+\frac{n}{\lambda}\right)^{-3}
$$

The modification of the viscosity in the Knudsen layer is also used in connection with a simple slip boundary condition, as given by Eq. (4), with $A=\sqrt{2 / \pi}$. This approach is expected to allow the CFD method to accurately model the velocity profile in the Knudsen layer. This boundary condition is referenced later as the type-3 slip condition.

The wall-function approach has only been evaluated for isothermal flow conditions that are usually encountered in microflows. Although there will be some change to the heat transfer rate coefficient (based on a constant Prandtl number), there might be other changes necessary to give the correct temperature profile in the Knudsen layer for the nonisothermal hypersonic flows considered here. Nevertheless, it is instructive to investigate the possible improvement this wall function might afford.

For all the slip boundary conditions, the one parameter that remains to be determined is the accommodation coefficient. The proper value used for the accommodation coefficient depends on many things, including the gas and the type of surface considered. For the purposes of this study, it is sufficient to ensure that both computational methods are consistent. Therefore, all solutions presented here are computed assuming a fully diffuse wall, with a corresponding accommodation coefficient of 1 .
The traditional no-slip boundary condition is referenced later as the type- 0 boundary condition.

\section{Results}

This investigation seeks to quantify the differences in the surface properties of a cylinder in a hypersonic flow by comparing CFD and DSMC predictions for total drag and peak heat transfer rate. Additionally, the overall flow features, such as the temperature field, are compared, as well as the surface distributions of pressure, shear stress, and heat transfer.

Tables 2 and $\underline{3}$ compare the total drag and the peak heat transfer rates predicted by both computational methods. Here, the peak heat transfer rate is obtained by averaging over the surface area within $1 \mathrm{deg}$ of the stagnation point. For CFD, these quantities are calculated for each of the different boundary conditions implemented.

It is clear that the slip boundary conditions improve the agreement between the two simulation methods. Although the difference in total drag is as high as 23.6 and $32.0 \%$ for the no-slip condition, the type-2 slip boundary condition shows the best agreement, with the difference exceeding $5 \%$ only for the $K n=0.25$ case. Similar trends are noted for the peak heat transfer rate, with a nearly $40 \%$ difference for the no-slip CFD solution, whereas the type-2 slip solution remains near $1 \%$ or less for most of the cases and only exceeds $5 \%$ for the $K n=0.25$ case at Mach 25 .

There is also a slight improvement in drag and heat flux agreement between the type- 1 and type- 3 boundary conditions, in which the only difference in the two boundary conditions is the inclusion in the type 3 of the viscosity correction function within the Knudsen layer. However, this improvement is not as great as that achieved with the type- 2 boundary condition.

The breakdown parameter is calculated using both the CFD and the DSMC solutions using Eq. (1), with the derivative being taken in the direction of the steepest gradient. For a cylinder in a hypersonic flow of a simple gas, the only causes of breakdown to the continuum hypothesis expected are in regions of high gradients (such as the shock and boundary layer) and regions of rarefaction (such as in the wake). The amount of continuum breakdown is also expected to increase as the gas flow becomes more rarefied. These trends are confirmed by the results shown next. In general, the flow experiences

Table 2 Total drag

\begin{tabular}{lccccc}
\hline \hline & \multicolumn{5}{c}{ Drag/length (\% difference), N/m } \\
\cline { 2 - 6 }$K n_{\infty}$ & DSMC & CFD 0 & CFD 1 & CFD 2 & CFD 3 \\
\hline & & Mach 10 & \\
0.002 & 187.6 & $187.5(-0.1 \%)$ & $187.4(-0.1 \%)$ & $187.4(-0.1 \%)$ & $187.6(0.0 \%)$ \\
0.01 & 40.02 & $40.32(0.8 \%)$ & $40.19(0.4 \%)$ & $40.16(0.4 \%)$ & $40.27(0.6 \%)$ \\
0.05 & 8.900 & $9.416(5.8 \%)$ & $9.122(2.5 \%)$ & $8.863(-0.4 \%)$ & $9.078(2.0 \%)$ \\
0.25 & 2.092 & $2.585(23.6 \%)$ & $2.301(10.0 \%)$ & $1.982(-5.2 \%)$ & $2.253(7.7 \%)$ \\
& & Mach 25 & & \\
0.002 & 1171 & $1176(0.4 \%)$ & $1176(0.4 \%)$ & $1176(0.4 \%)$ & $1177(0.5 \%)$ \\
0.01 & 250.8 & $255.3(1.8 \%)$ & $254.6(1.5 \%)$ & $254.4(1.4 \%)$ & $255.1(1.7 \%)$ \\
0.05 & 56.85 & $61.17(7.6 \%)$ & $59.38(4.5 \%)$ & $57.04(0.3 \%)$ & $58.90(3.6 \%)$ \\
0.25 & 13.35 & $17.61(32.0 \%)$ & $15.73(17.8 \%)$ & $13.02(-2.4 \%)$ & $15.14(13.4 \%)$ \\
\hline \hline
\end{tabular}

Table 3 Peak heat transfer rate

\begin{tabular}{lccccc}
\hline \hline & \multicolumn{5}{c}{ Peak heating (\% difference), $\mathrm{kW} / \mathrm{m}^{2}$} \\
\cline { 2 - 6 }$K n_{\infty}$ & DSMC & CFD 0 & CFD 1 & CFD 2 & CFD 3 \\
\hline \multirow{5}{*}{0.002} & 89.80 & $89.85(0.0 \%)$ & $89.14(-0.7 \%)$ & $89.00(-0.9 \%)$ & $88.54(-1.4 \%)$ \\
0.01 & 39.13 & $40.22(2.8 \%)$ & $39.49(0.9 \%)$ & $39.20(0.2 \%)$ & $38.89(-0.6 \%)$ \\
0.05 & 15.92 & $18.08(13.6 \%)$ & $17.25(8.4 \%)$ & $15.79(-0.8 \%)$ & $16.81(5.6 \%)$ \\
0.25 & 5.926 & $7.851(32.5 \%)$ & $7.061(19.1 \%)$ & $6.184(4.3 \%)$ & $6.640(12.1 \%)$ \\
& & Mach 25 & & \\
0.002 & 1746 & $1763(0.9 \%)$ & $1750(0.2 \%)$ & $1746(0.0 \%)$ & $1739(-0.4 \%)$ \\
0.01 & 749.6 & $791.0(5.5 \%)$ & $777.4(3.7 \%)$ & $768.4(2.5 \%)$ & $765.3(2.1 \%)$ \\
0.05 & 309.9 & $357.9(13.6 \%)$ & $341.0(10.4 \%)$ & $294.2(-4.8 \%)$ & $331.2(7.2 \%)$ \\
0.25 & 105.7 & $147.8(39.9 \%)$ & $133.6(19.1 \%)$ & $111.7(5.7 \%)$ & $123.7(17.0 \%)$ \\
\hline \hline
\end{tabular}


continuum breakdown in three areas; across the bow shock, in the boundary layer and in the wake region. The flow in the shock and boundary-layer regions experiences very steep gradients in flow properties, whereas the wake region is more rarefied, thus leading to the breakdown of the continuum hypothesis.

In the results that follow, the surface properties are presented in terms of nondimensional coefficients with the following definitions:

$$
\begin{gathered}
C_{P}=\frac{p-p_{\infty}}{\frac{1}{2} \rho_{\infty} U_{\infty}^{2}} \\
C_{F}=\frac{\tau}{\frac{1}{2} \rho_{\infty} U_{\infty}^{2}} \\
C_{H}=\frac{q}{\frac{1}{2} \rho_{\infty} U_{\infty}^{3}}
\end{gathered}
$$

where $p$ is the pressure, $\tau$ is the shear stress, $q$ is the heat transfer rate, $p_{\infty}$ is the freestream pressure, $\rho_{\infty}$ is the freestream density, and $U_{\infty}$ is the freestream velocity. The surface properties in each case are plotted as a function of the angle around the cylinder, with the stagnation point being located at an angle of zero (see Fig. 1).

Along with the surface properties, the maximum $K n_{\mathrm{GLL}}$ at the surface (based on the CFD solution with type- 2 slip conditions) is also plotted in each case.

$$
K \boldsymbol{n}=\mathbf{0 . 0 0 2}
$$

At a Knudsen number of 0.002 , the flow is within the continuum (no-slip) regime. Nevertheless, there is still evidence of continuum breakdown in the shock, along the cylinder surface in the boundary layer, and in the wake, as seen in Fig. 2. Interestingly, DSMC predicts a larger degree of breakdown than does CFD. The increase in velocity from Mach 10 to Mach 25 also slightly increases the amount of breakdown predicted in the shock and wake regions.

The temperature fields predicted by CFD and DSMC, as seen in Fig. 3, are in excellent agreement. The Mach-25 case shows an increase in the maximum temperature as well as a slight increase in the shock thickness; however, for the presently considered simple gas, there are no other effects. The surface properties shown in Figs. 4-6 show very good agreement between the two methods for all types of CFD boundary conditions considered and for both Mach 10 and Mach 25 . The only location along the surface on which $K n_{\mathrm{GLL}}$ is greater than 0.05 is in the wake region. The total drag and peak heat transfer rates predicted by the two methods differ by less than $1 \%$, with only one exception (that being the type-3 CFD solution at $1.4 \%$ ). These results are well within the error range expected for a flow for which both methods are valid.

$$
K n=0.01
$$

A Knudsen number of 0.01 is considered to be near the limit of the continuum (no-slip) regime. Here, there is increased evidence of continuum breakdown, as shown in Fig. 7. However, general flowfield features still show good agreement. The temperature fields, seen in Fig. $\underline{8}$, are very similar, with a few exceptions in the shock thickness and the wake (for Mach 25), in which the continuum hypothesis is expected to break down first. The shock standoff distance predicted by both methods is nearly the same for the Mach10 case, although there is more of a difference at Mach 25. The

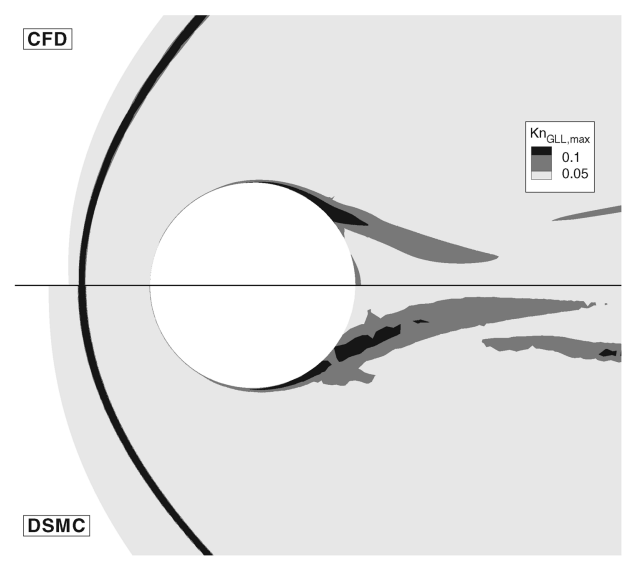

a) Mach 10

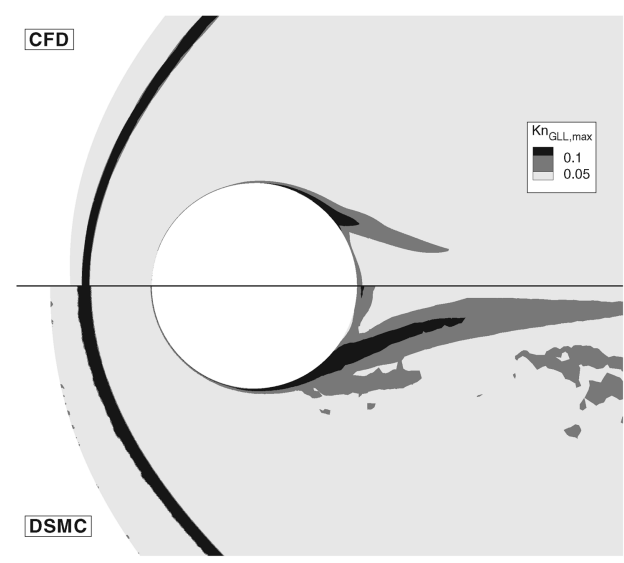

b) Mach 25

Fig. $2 K n_{\infty}=0.002 K n_{\mathrm{GLL}}$ for Mach 10 and Mach 25; light gray regions correspond to $K n_{\mathrm{GLL}}<0.05$, dark gray regions correspond to $K n_{\mathrm{GLL}}<0.10$, and black regions correspond to $K n_{\mathrm{GLL}}>0.10$; continuum breakdown is predicted in the shock region, in a thin boundary layer along the surface, and in a region of flow expansion around the top of the cylinder.

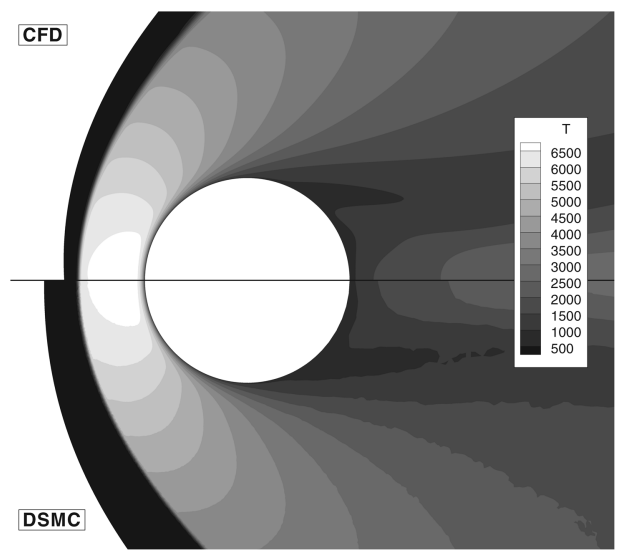

a) Mach 10

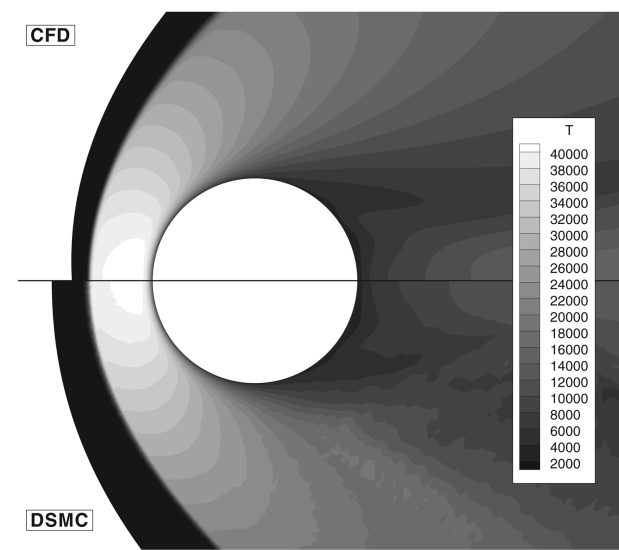

b) Mach 25

Fig. $3 K n_{\infty}=0.002$ temperature field (in Kelvin) for Mach 10 and Mach 25; CFD is shown with a type-2 boundary condition. 


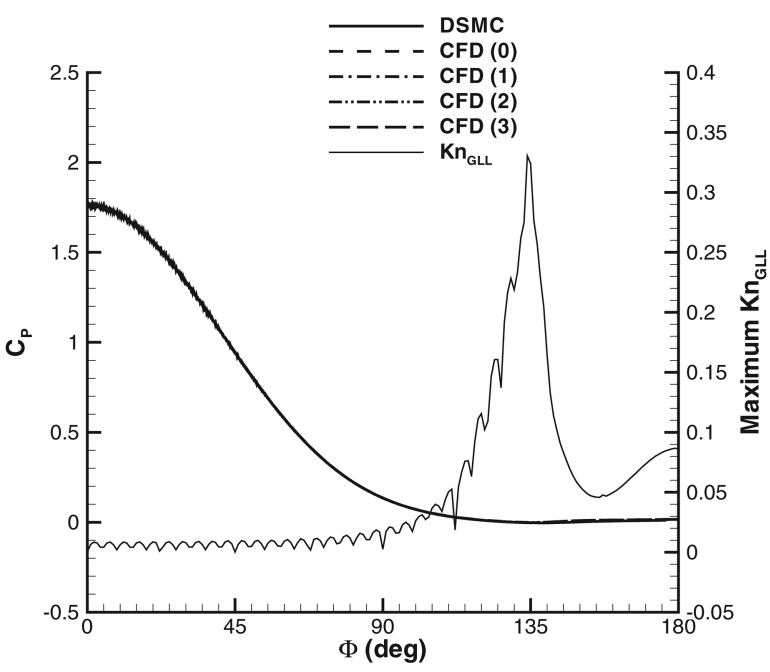

a) Mach 10

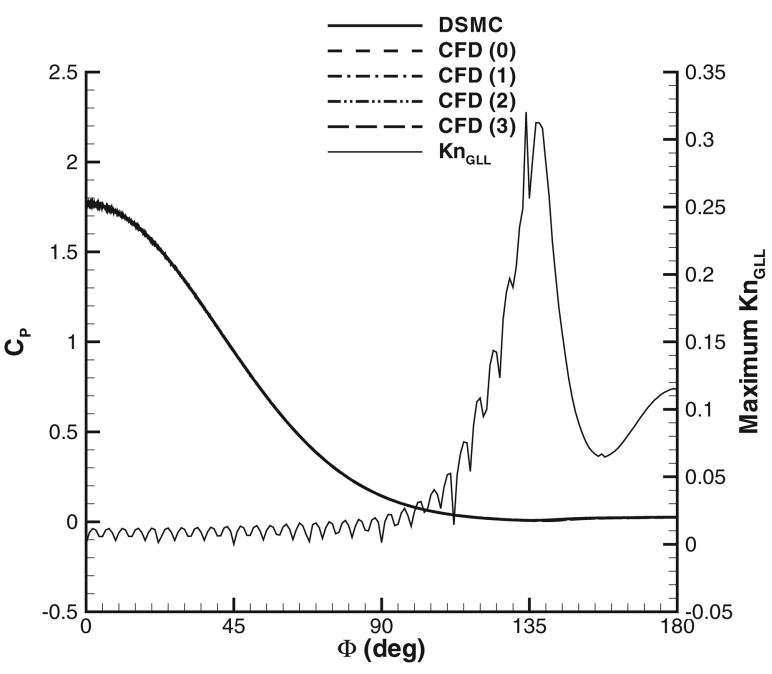

b) Mach 25

Fig. $4 K n_{\infty}=0.002$ surface pressure coefficient and $K n_{\mathrm{GLL}}$ for Mach 10 and Mach 25 .

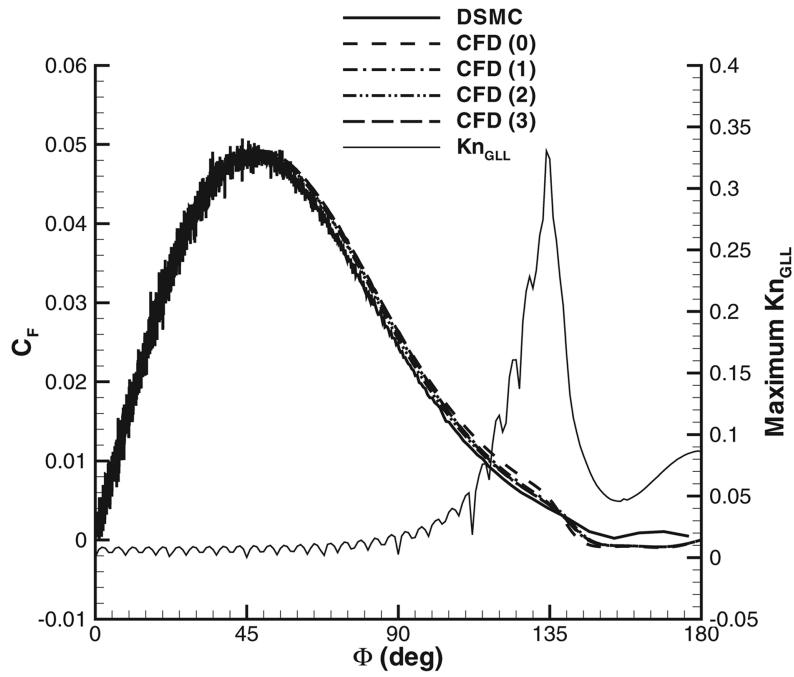

a) Mach 10

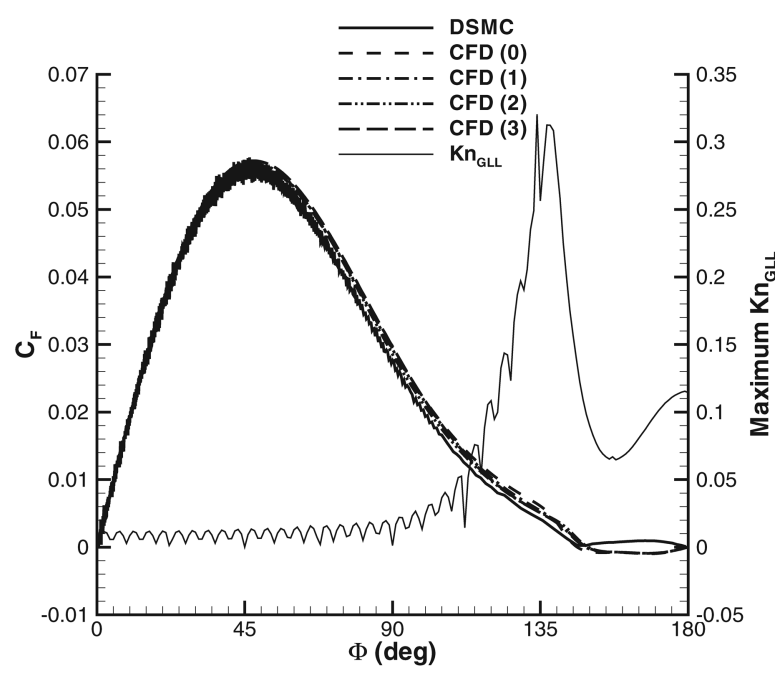

b) Mach 25

Fig. $5 K n_{\infty}=0.002$ surface friction coefficient and $K n_{\text {GLL }}$ for Mach 10 and Mach 25.

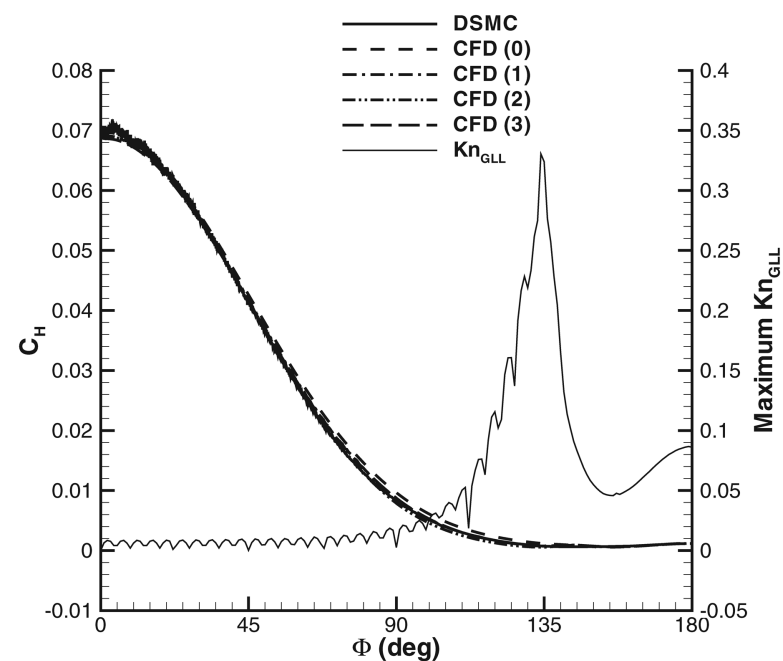

a) Mach 10

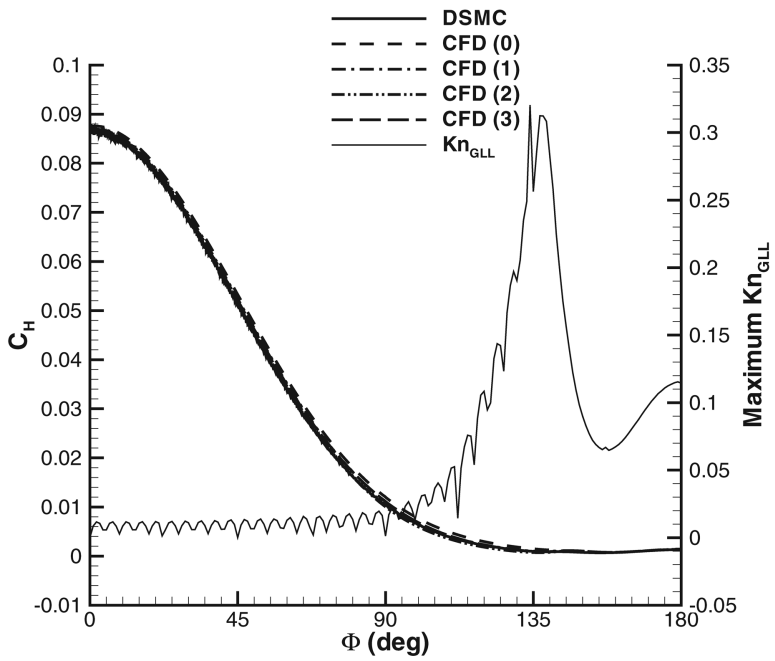

b) Mach 25

Fig. $6 K n_{\infty}=0.002$ surface heating coefficient and $K n_{\mathrm{GLL}}$ for Mach 10 and Mach 25 . 


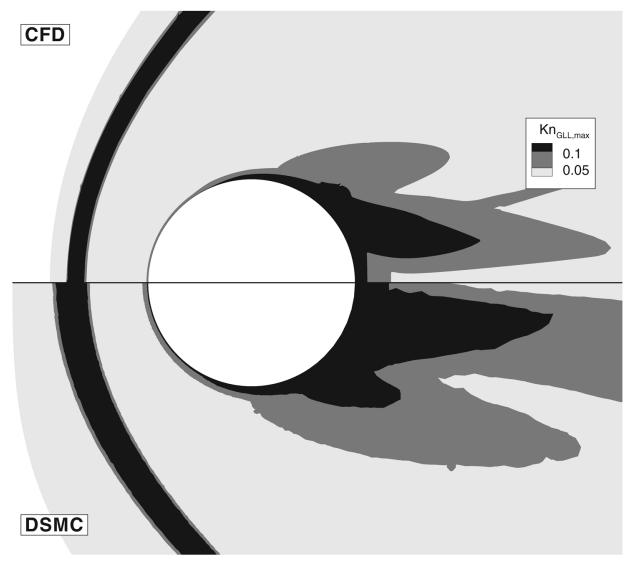

a) Mach 10

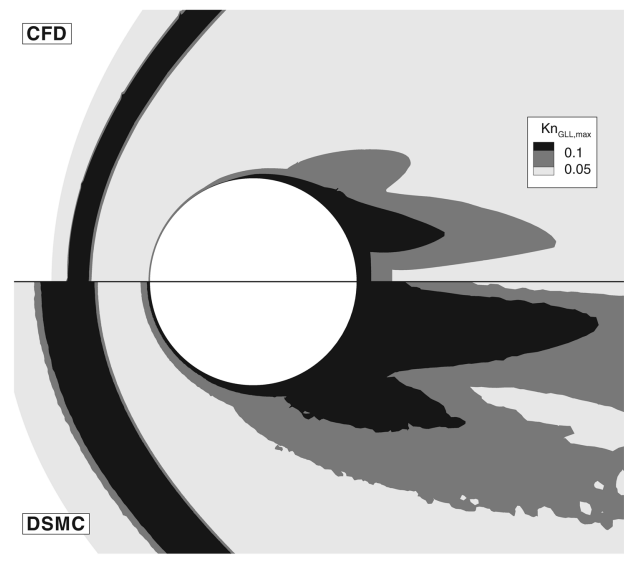

b) Mach 25

Fig. $7 K n_{\infty}=0.01 K n_{\mathrm{GLL}}$ for Mach 10 and Mach 25; light gray regions correspond to $K n_{\mathrm{GLL}}<0.05$, dark gray regions correspond to $K n_{\mathrm{GLL}}<0.10$, and black regions correspond to $K n_{\mathrm{GLL}}>\mathbf{0 . 1 0}$. There are larger regions in which continuum breakdown becomes a concern, especially in the wake, the shock, and the boundary layer. The Mach-25 case shows larger regions than the Mach-10 case.

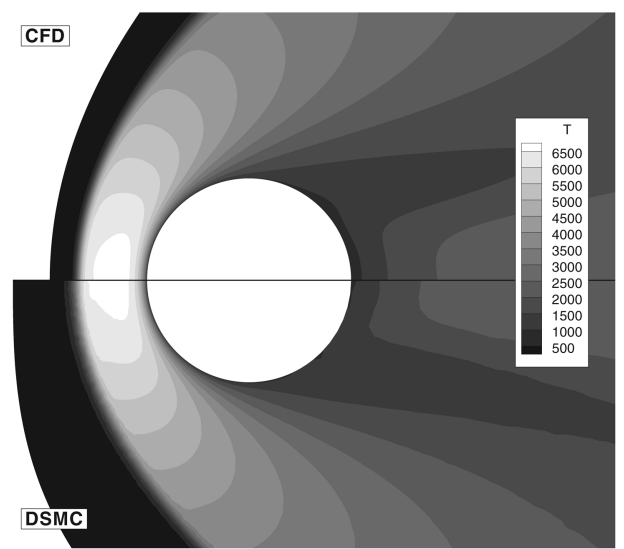

a) Mach 10

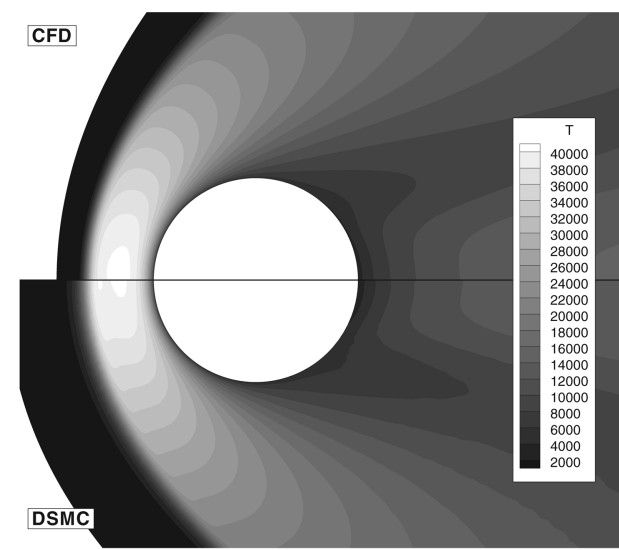

b) Mach 25

Fig. $8 K n_{\infty}=0.01$ temperature field (in Kelvin) for Mach 10 and Mach 25; CFD is shown with a type-2 boundary condition.
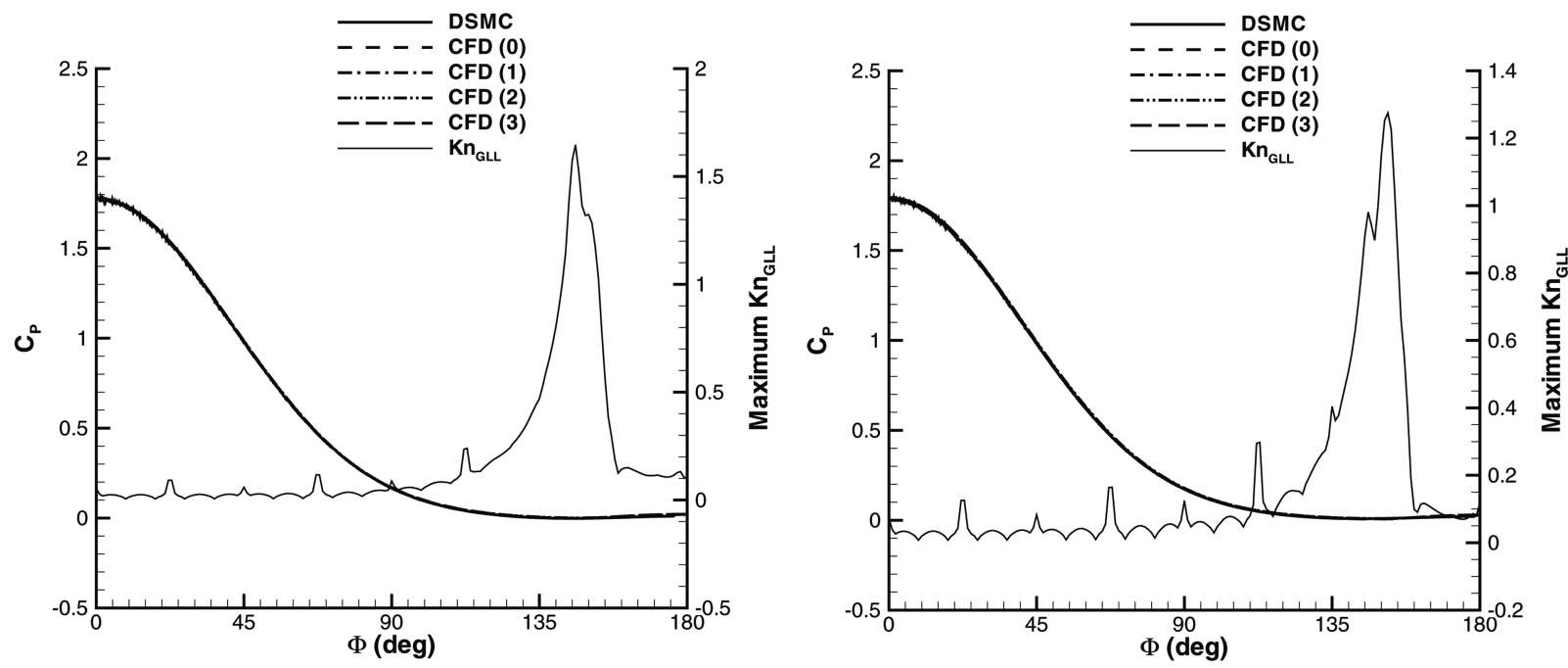

a) Mach 10

b) Mach 25

Fig. $9 K n_{\infty}=0.01$ surface pressure coefficient and $K n_{\text {GLL }}$ for Mach 10 and Mach 25 .

thicker shock does not seem to have an effect on the surface properties, as shown later.

The amount of breakdown in the shock does not necessarily carry over to the surface properties. Again, the amount of continuum breakdown near the surface does not seem to significantly affect the surface properties, shown in Figs. 9-11. The no-slip CFD case (type0 ) shows some divergence from the others in the wake. The best agreement is achieved with the type- 2 and type- 3 slip conditions. Shear stress starts to diverge, even with the slip conditions, at Mach 25. This particular case seems to be somewhat of an aberration, 


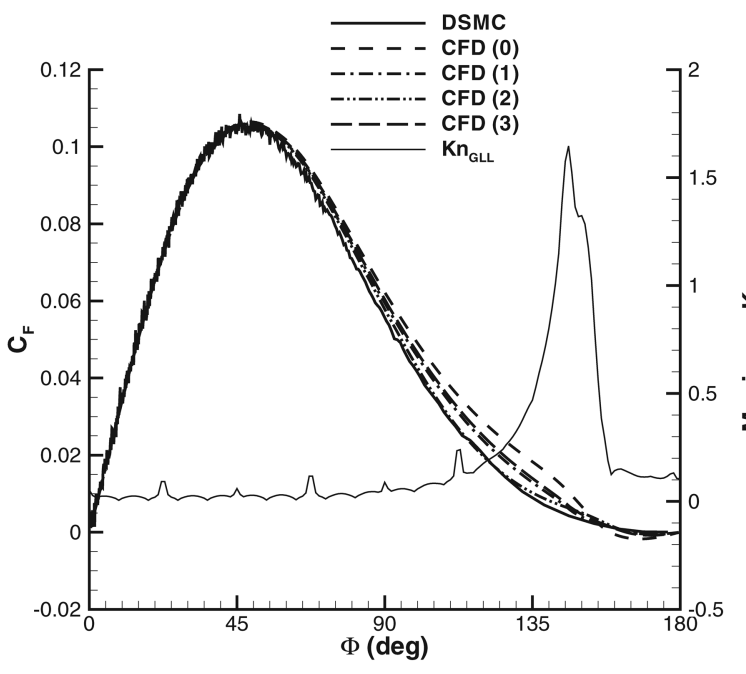

a) Mach 10

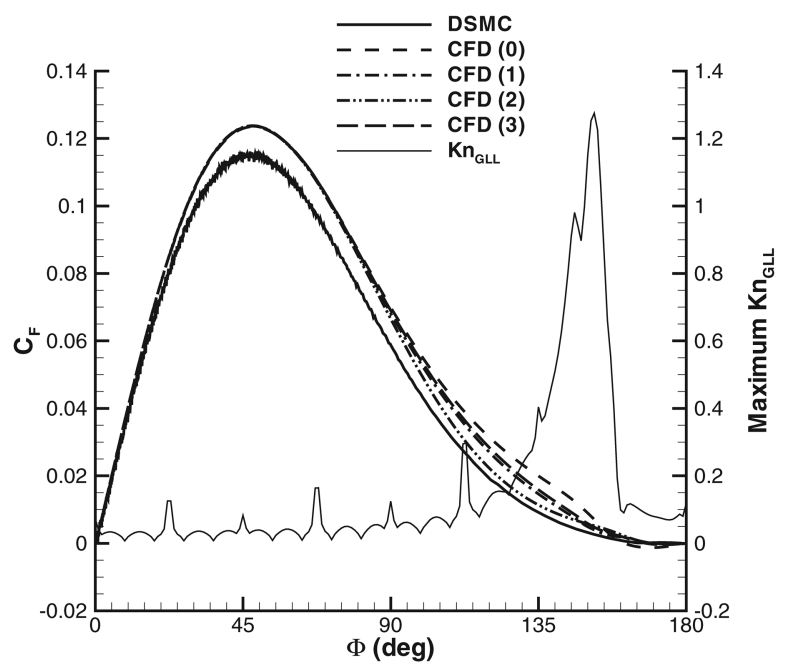

b) Mach 25

Fig. $10 K n_{\infty}=0.01$ surface friction coefficient and $K n_{\text {GLL }}$ for Mach 10 and Mach 25 .

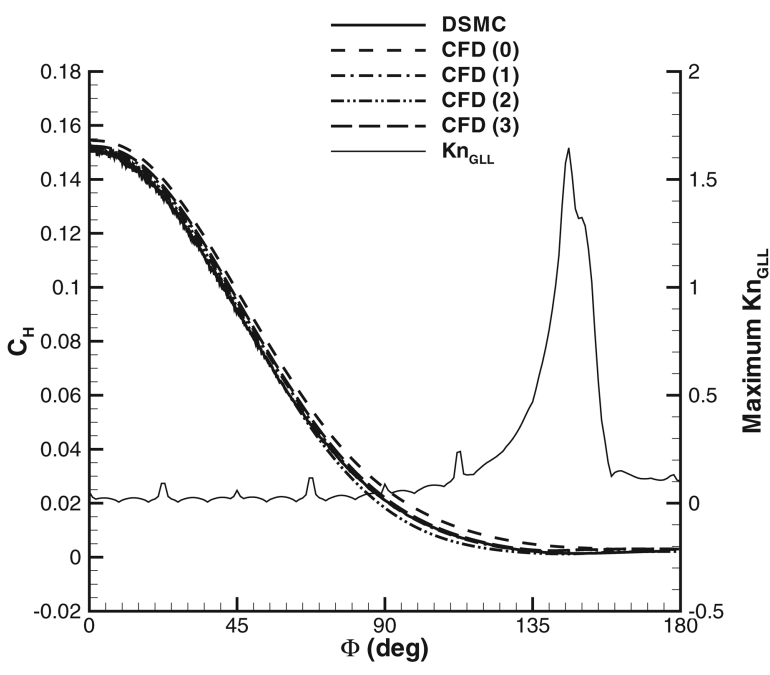

a) Mach 10

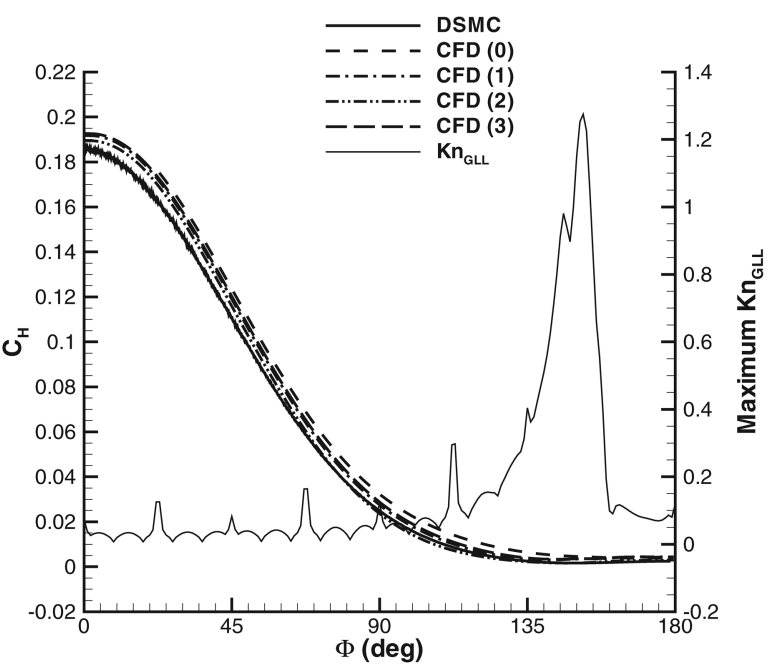

b) Mach 25

Fig. $11 K n_{\infty}=0.01$ surface heating coefficient and $K n_{\text {GLL }}$ for Mach 10 and Mach 25 .

especially when considering the good agreement that will be shown next for the $K n=0.05$ case. The heating rates also agree well at Mach 10, but show some differences at the higher velocities of Mach 25 . The total drag due to pressure and viscous effects predicted by CFD is still within $1 \%$ of that predicted by DSMC for Mach 10 and within 2\% for Mach 25, as shown in Table 2. The peak heating also differs by less than $2 \%$ for the Mach- 10 case when using slip boundary conditions and by less than $3 \%$ for the best slip conditions (type 2 and type 3 ) for the Mach-25 case. These results indicate that the surface properties are not strongly affected by the continuum breakdown in the shock, and the slip conditions improve the agreement quite satisfactorily.

$$
K \boldsymbol{n}=0.05
$$

At a Knudsen number of 0.05 , the flow is well within the slip regime. The flow demonstrates breakdown in a larger area of the flow in each of the three regions (shock, boundary layer, and wake), as seen in Fig. 12. Again, there are larger areas in the Mach-25 case in which the breakdown parameter exceeds the critical value of 0.05 ; in fact, nearly the entire domain is predicted to be beyond the region of applicability of the continuum equations. The differences between the CFD and DSMC temperature fields shown in Fig. 13 are more pronounced than the lower-Knudsen-number cases, with even more differences for the higher-Mach-number case. The DSMC shock is much thicker than the CFD shock, although the shock standoff distance and peak temperatures are still very nearly equal. In the wake, DSMC predicts a higher temperature than CFD.

The surface pressure predicted by both methods is still in excellent agreement, as seen in Fig. 14. However, the shear stress is higher for most of the CFD cases, especially in the wake, with the surprising exception of the type-2 case, which agrees almost exactly with the DSMC case (see Fig. 15). The total drag for all of the slip cases is within $5 \%$ of the DSMC case, with the type- 2 case being almost identical. The heat transfer rate, shown in Fig. 16, also shows surprisingly good agreement between the type-2 case and DSMC. Although the no-slip case predicts a peak heat transfer rate about $13 \%$ higher than DSMC, the slip cases show better agreement, with the type- 2 case within $1 \%$ for Mach 10 and less than 5\% for Mach 25.

$$
K \boldsymbol{n}=\mathbf{0 . 2 5}
$$

For $K n_{\infty}=0.25$, the flow is considered outside of the slip regime and into the transition regime. Here, even the addition of slip boundary conditions is not expected to help the continuum CFD method's predictive capabilities very much. Indeed, the plots of the breakdown parameter in Fig. 17 indicate that there are significant nonequilibrium effects across almost all of the flow domain. The temperature fields predicted by both methods, seen in Fig. 18, show some major differences. Although the peak temperatures behind the 


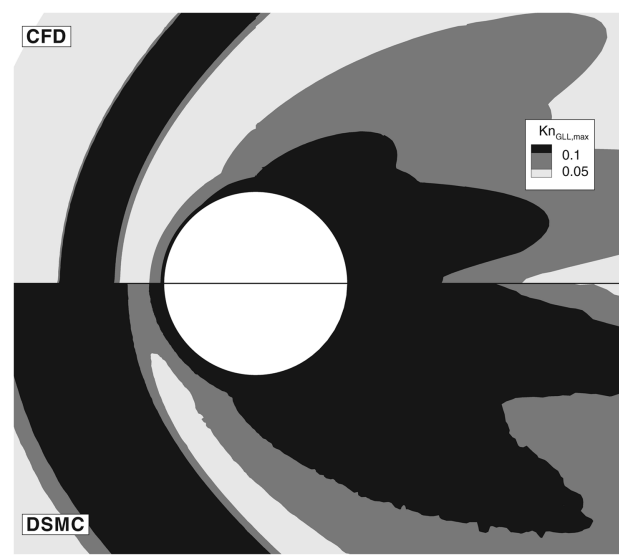

a) Mach 10

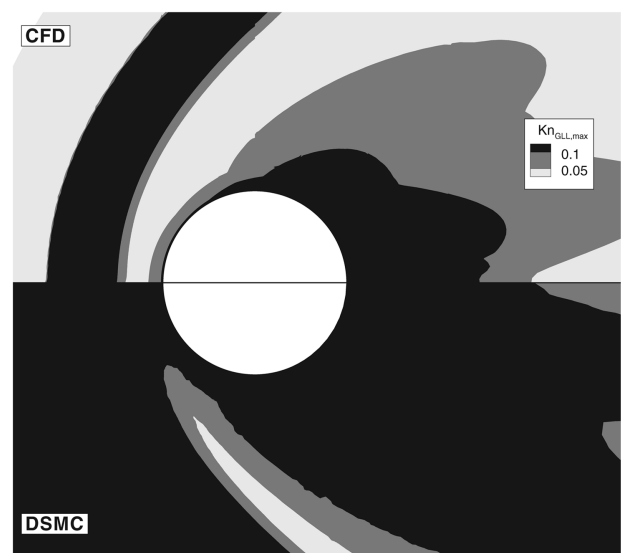

b) Mach 25

Fig. $12 K n_{\infty}=0.05 K n_{\mathrm{GLL}}$ for Mach 10 and Mach 25; light gray regions correspond to $K n_{\mathrm{GLL}}<0.05$, dark gray regions correspond to $K n_{\mathrm{GLL}}<0.10$, and black regions correspond to $K n_{\mathrm{GLL}}>\mathbf{0 . 1 0}$. Continuum breakdown is predicted in much larger regions than in previous cases. The DSMC method predicts larger regions in which continuum breakdown exceeds the critical value of 0.05 , with an even larger region predicted for the Mach-25 case.

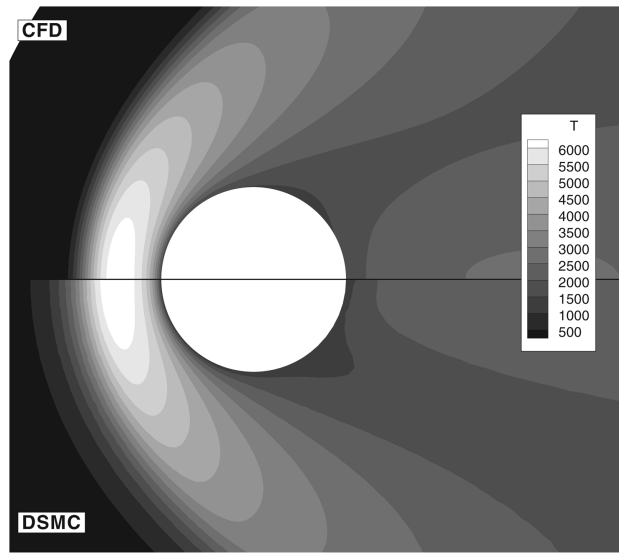

a) Mach 10

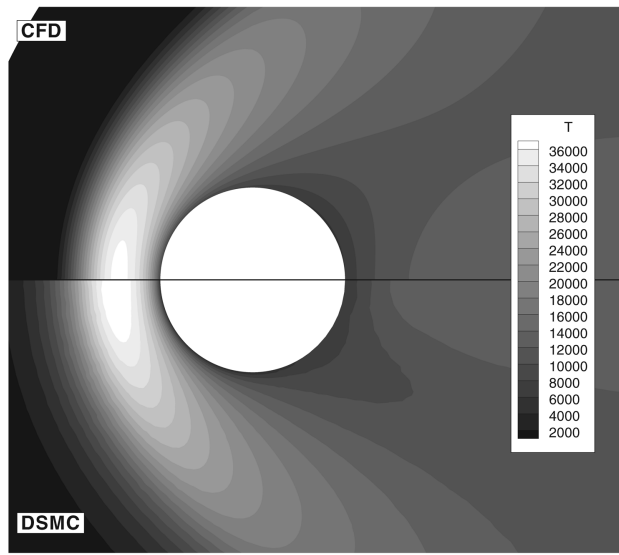

b) Mach 25

Fig. $13 K n_{\infty}=0.05$ temperature field (in Kelvin) for Mach 10 and Mach 25; CFD is shown with a type-2 boundary condition.

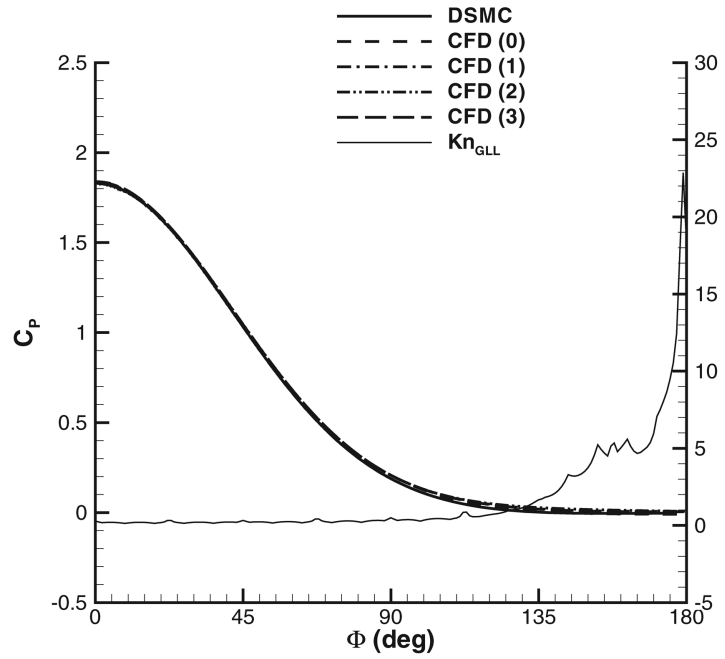

a) Mach 10

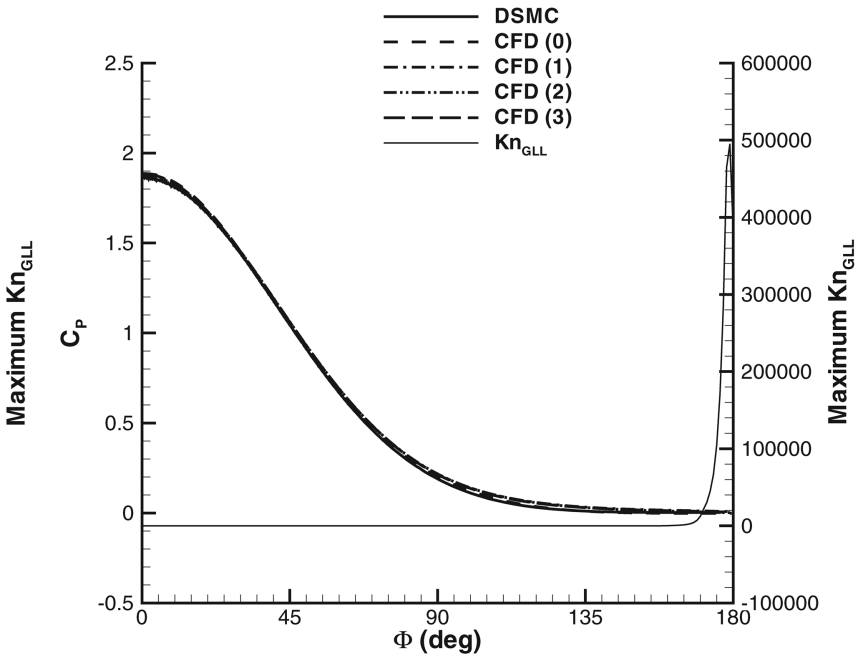

b) Mach 25

Fig. $14 K n_{\infty}=0.05$ surface pressure coefficient and $K n_{\text {GLL }}$ for Mach 10 and Mach 25 .

shock appear to be about the same, the shock standoff distances are significantly different, as is the thermal boundary-layer thickness.

The surface pressure, plotted in Fig. 19, predicted by both methods is also no longer in agreement, even for the CFD cases implementing slip conditions. The DSMC pressure is less than the CFD pressure near the forebody (most likely due to the thinner shock predicted by CFD, which allows the CFD solution to approach the ideal jump condition more closely than the DSMC solution), but the agreement does improve in the wake. The pressure tensor at this Knudsen number is also most likely nonisotropic, which would introduce 


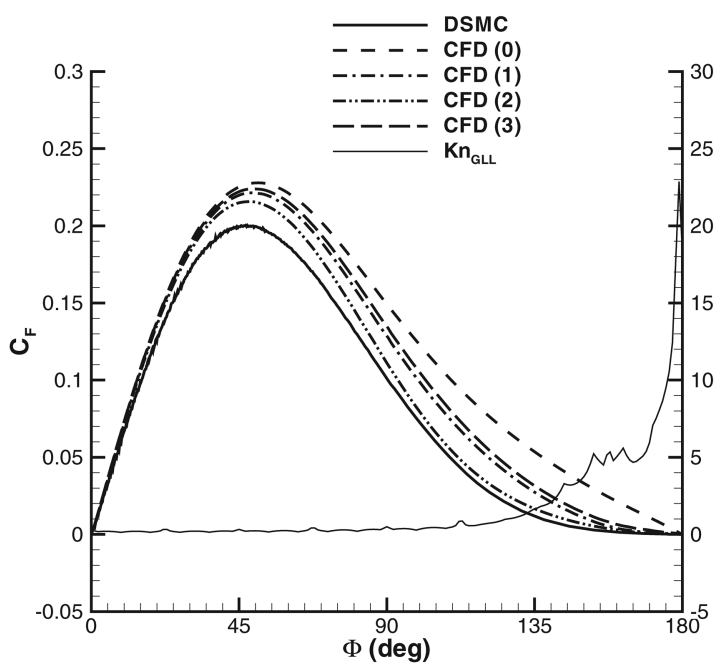

a) Mach 10

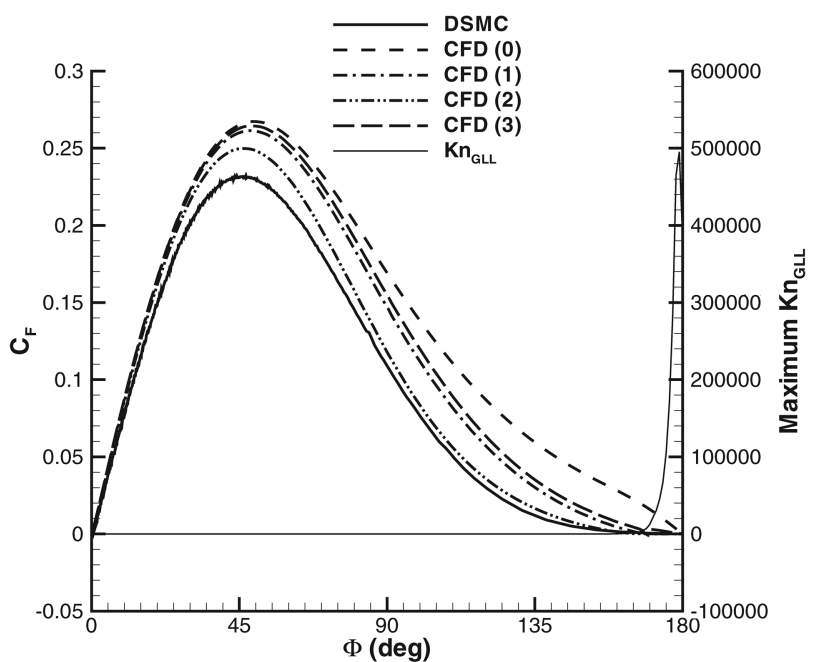

b) Mach 25

Fig. $15 K n_{\infty}=0.05$ surface friction coefficient and $K n_{\text {GLL }}$ for Mach 10 and Mach 25.

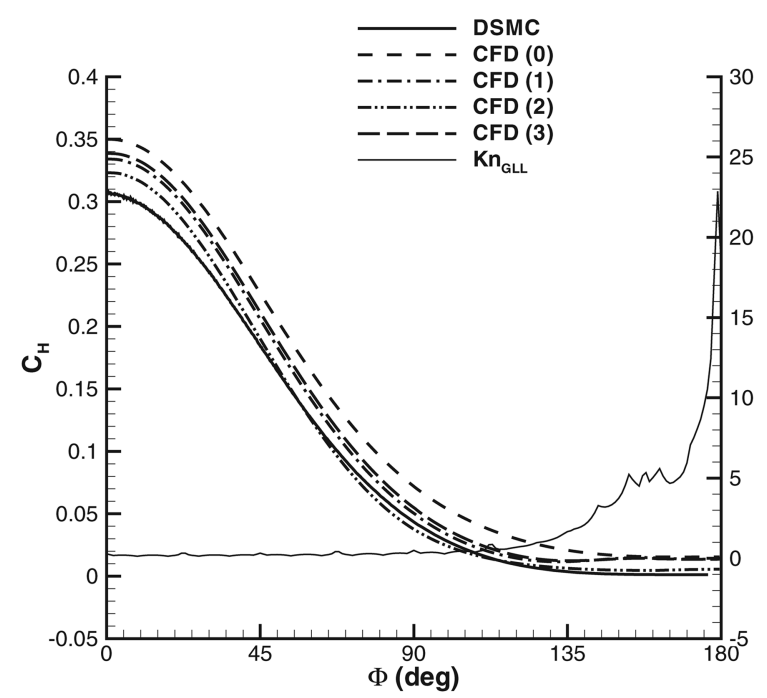

a) Mach 10

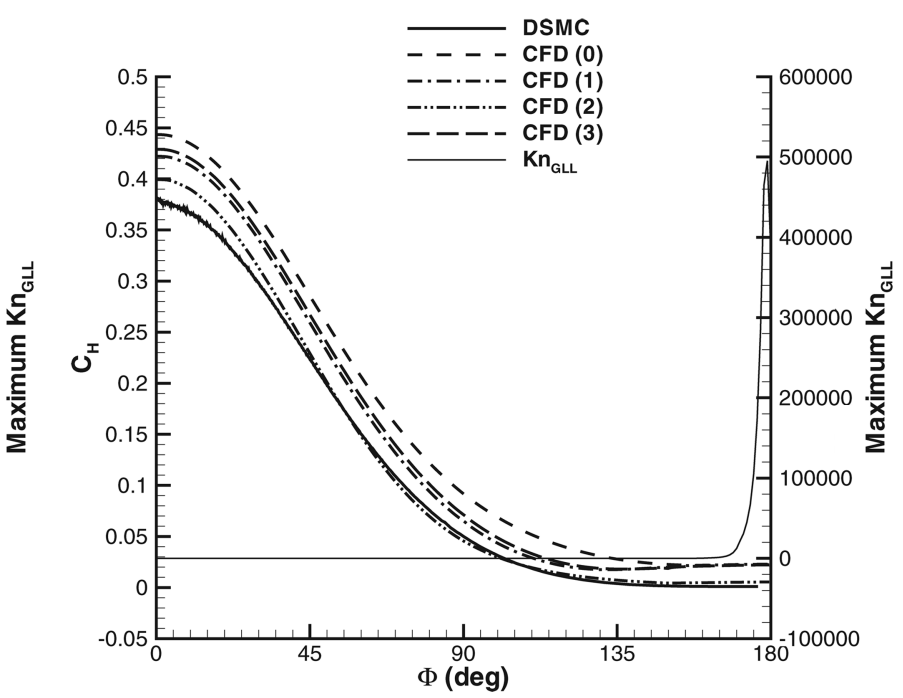

b) Mach 25

Fig. $16 K n_{\infty}=0.05$ surface heating coefficient and $K n_{\text {GLL }}$ for Mach 10 and Mach 25 .

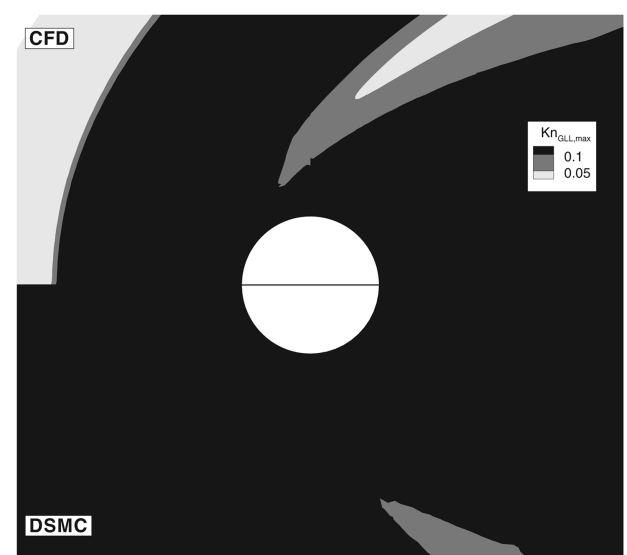

a) Mach 10

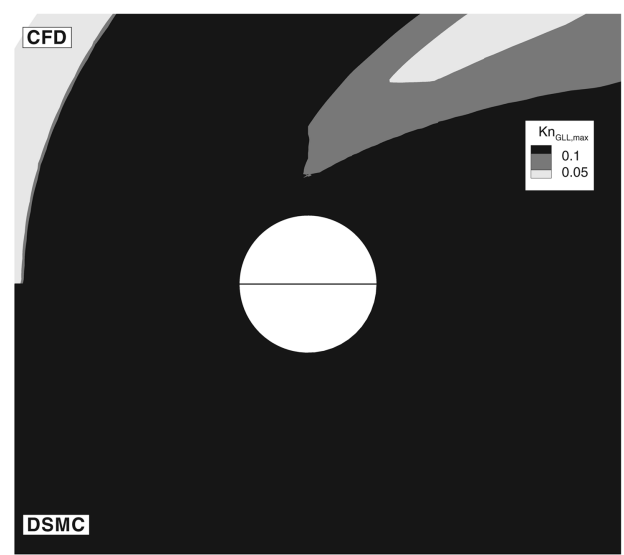

b) Mach 25

Fig. $17 K n_{\infty}=0.25 K n_{\mathrm{GLL}}$ for Mach 10 and Mach 25; light gray regions correspond to $K n_{\mathrm{GLL}}<0.05$, dark gray regions correspond to $K n_{\mathrm{GLL}}<0.10$, and black regions correspond to $K n_{\mathrm{GLL}}>\mathbf{0 . 1 0}$; continuum breakdown is predicted in almost the entire domain surrounding the cylinder.

errors into the CFD solution. The shear stress, shown in Fig. 20, shows the same general trend as in previous cases in that both methods agree near the stagnation region, but the results diverge as the flow accelerates around the cylinder. Here, the type-2 CFD solution predicts a lower shear stress, but a higher pressure, thus the overall drag predictions are somewhat close (differing by about $5 \%$ for Mach 10 and less than 3\% for Mach 25). The shear stress results also seem to indicate that the type- 2 CFD solution predicts some 


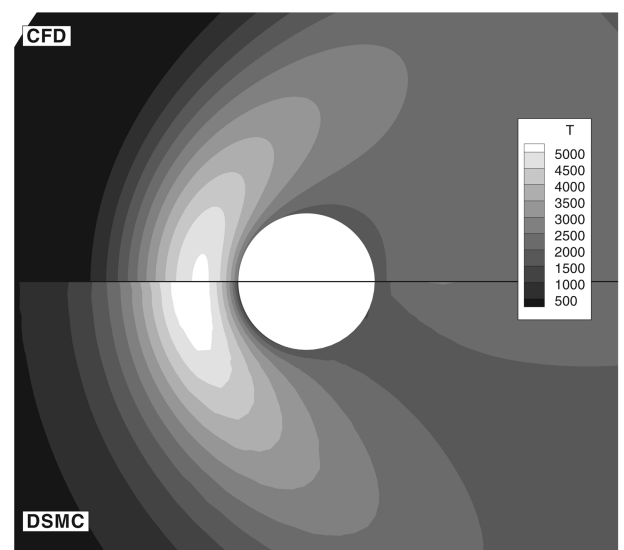

a) Mach 10

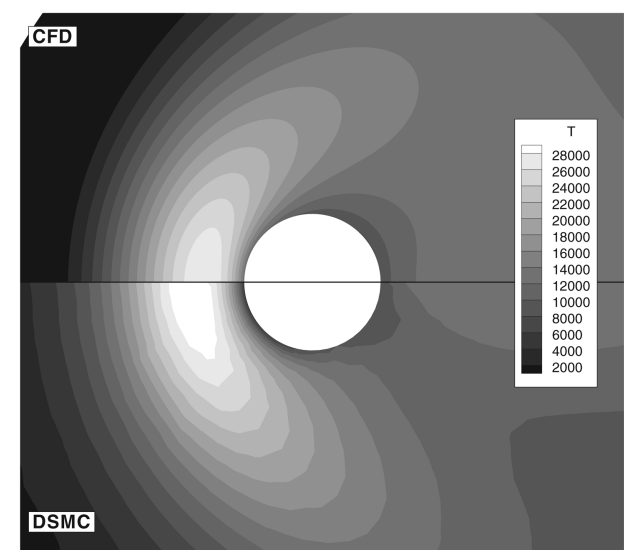

b) Mach 25

Fig. $18 K n_{\infty}=0.25$ temperature field (in Kelvin) for Mach 10 and Mach 25; CFD is shown with a type-2 boundary condition.

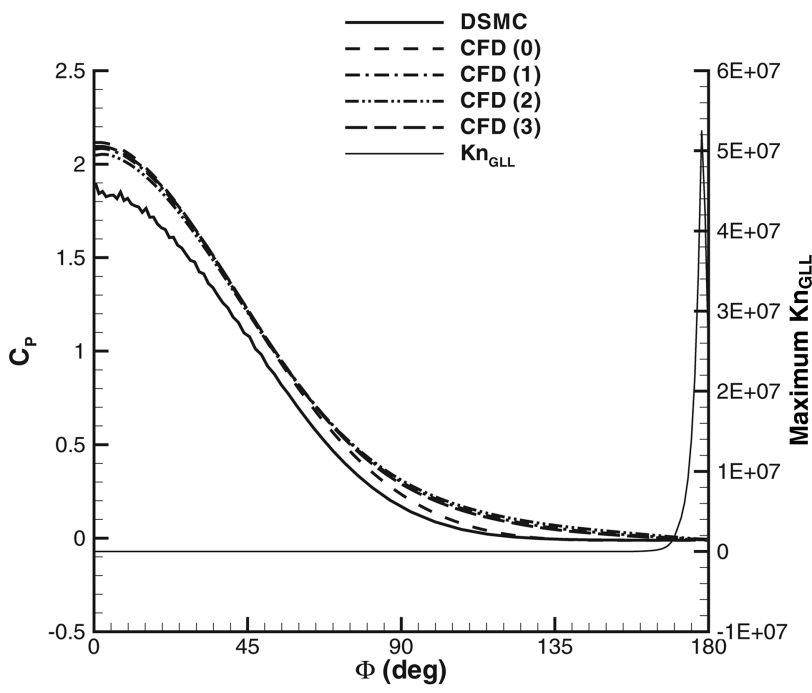

a) Mach 10

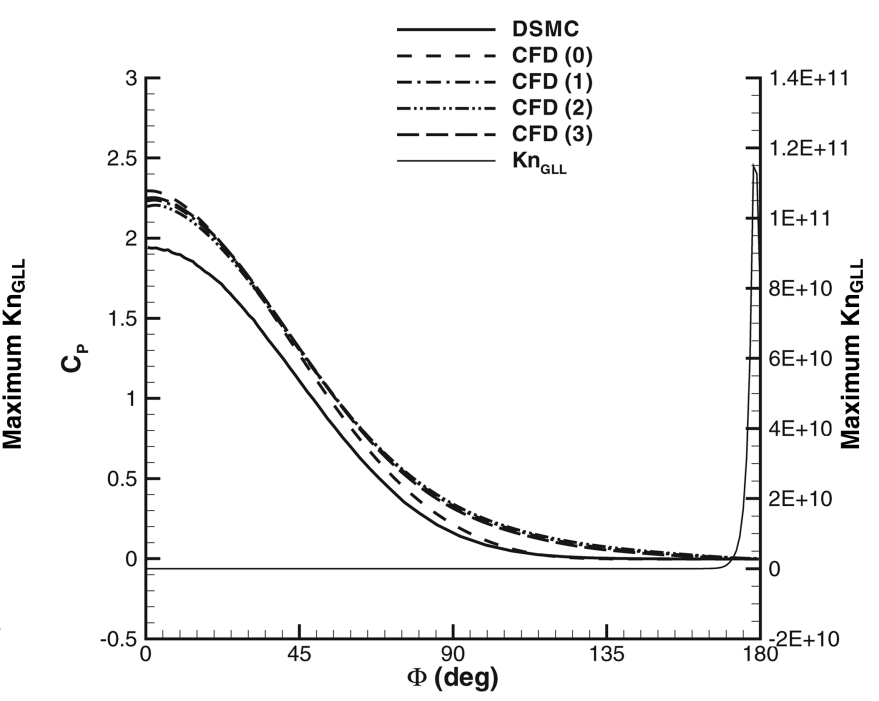

b) Mach 25

Fig. $19 K n_{\infty}=0.25$ surface pressure coefficient and $K n_{\text {GLL }}$ for Mach 10 and Mach 25.

separation in the wake that is not predicted by the other models. The heat transfer rate, as seen in Fig. 21, follows trends similar to the previous cases in that the DSMC heat transfer rate is lower than the CFD rate along the entire surface for all but the type- 2 case, which gets fairly close in some areas. The peak heat transfer rates differ by as much as almost $40 \%$ for the no-slip case at Mach 25, but the type-2 case shows the best agreement, with less than 5\% difference at Mach 10 and less than $6 \%$ difference at Mach 25.

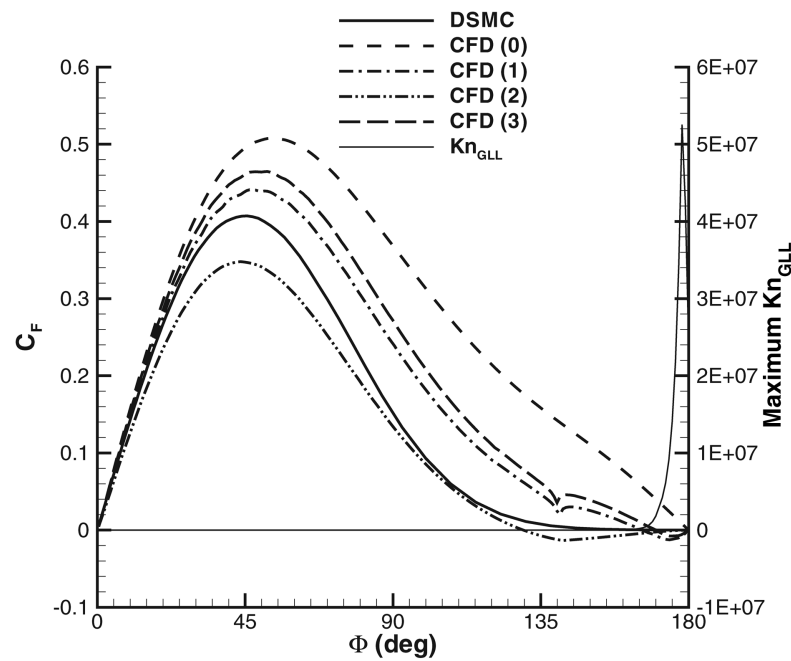

a) Mach 10

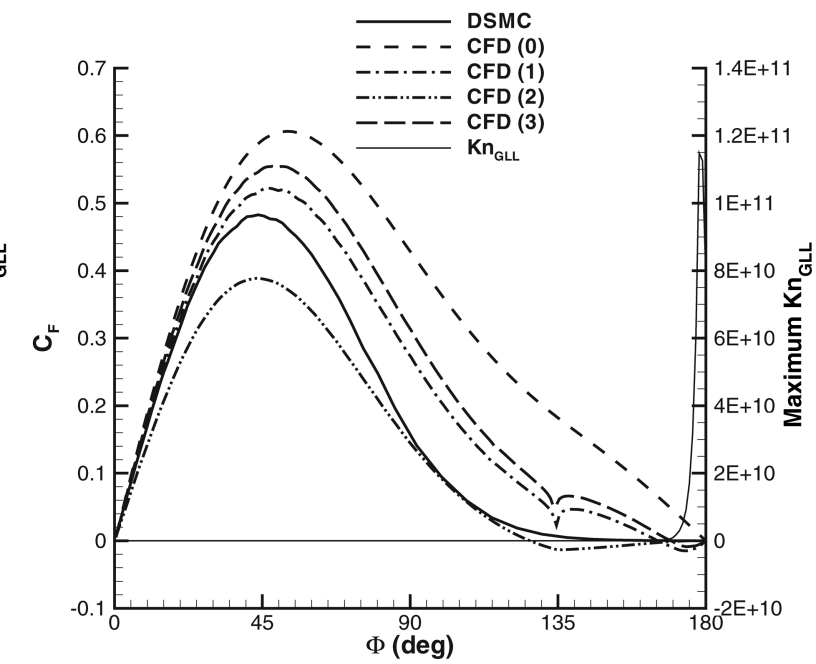

b) Mach 25

Fig. $20 K n_{\infty}=0.25$ surface friction coefficient and $K n_{\text {GLL }}$ for Mach 10 and Mach 25 . 


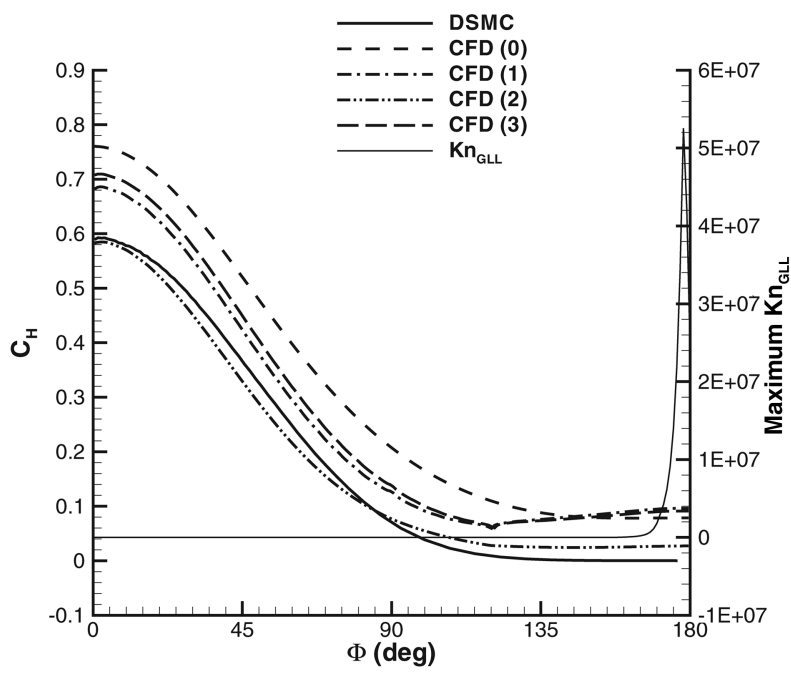

a) Mach 10

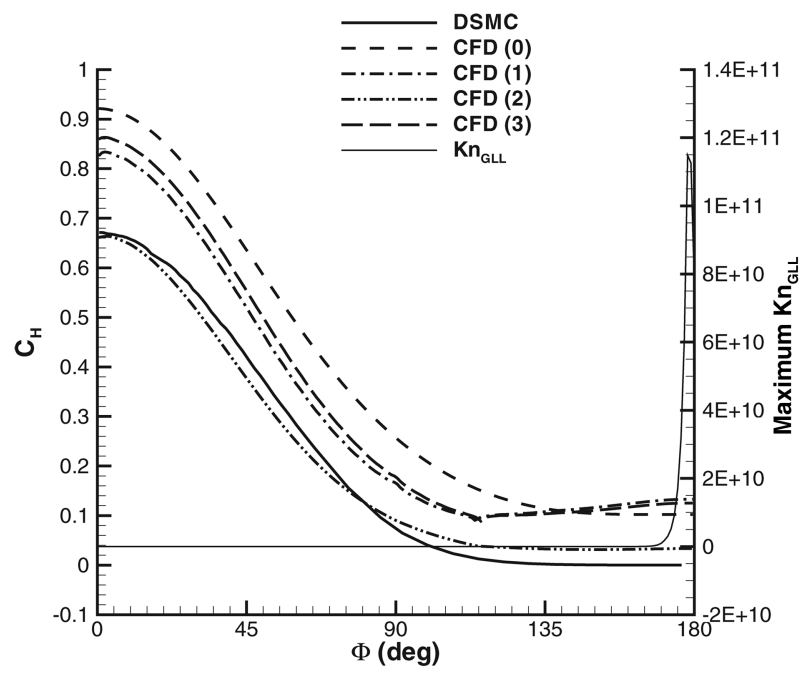

b) Mach 25

Fig. $21 K n_{\infty}=0.25$ surface heating coefficient and $K n_{\mathrm{GLL}}$ for Mach 10 and Mach 25.

\section{Conclusions}

The primary goal of this study was to quantify the difference between DSMC and CFD simulations when using slip boundary conditions, to determine the effects of different levels of continuum breakdown on surface properties under hypersonic flow conditions.

Comparison of CFD and DSMC results for identical flow conditions showed that the surface properties of pressure, shear stress, and heat transfer rates were very similar for the lowerKnudsen-number flows in which the continuum hypothesis is valid, as expected, whereas the results diverged in the higher-Knudsennumber cases. The surface pressure was least affected by continuum breakdown, as quantified by the gradient-length local Knudsen number, among those properties investigated and seemed to be affected only by nonequilibrium effects for the highest Knudsen number flow. The shear stress was most influenced by continuum breakdown and was affected primarily by continuum breakdown in the wake. The addition of velocity-slip and temperature-jump boundary conditions greatly improved the agreement at higher Knudsen numbers. Several different types of slip boundary conditions were examined, and the best agreement appeared to be obtained when using the generalized slip conditions proposed by Gökçen $[15,16]$. With these boundary conditions, the difference in surface properties predicted by CFD and DSMC increased from less than $1 \%$ at $K n_{\infty}=0.002$ to around $5 \%$ at $K n_{\infty}=0.25$.

For the case of a simple gas, the higher velocities associated with a Mach-25 flow did not increase the difference between the CFD and DSMC predictions by much. Although the extent of the region in which the continuum breakdown parameter exceeded the critical value of 0.05 was larger at the higher Mach number, the predicted surface properties with the slip boundary conditions still remained well under $5 \%$ for all but the $K n_{\infty}=0.25$ case, in which the peak heat transfer rates and total drag predictions were within $6 \%$.

There is, then, a clear advantage to the use of slip boundary conditions when using CFD models to predict the surface properties of a hypersonic vehicle. In fact, if one is interested only in predicting the drag of the vehicle, any of the slip boundary conditions will give results within $5 \%$ for a Knudsen number up to 0.05 . Accurate peak heating predictions (within 5-6\%) will require the use of the Gökçentype boundary condition. The choice of slip boundary condition is an important consideration not only regarding the accuracy of the flow predictions, but also regarding the computational expense of the simulations. All of the slip boundary conditions increased the computational expense of the CFD simulations, with the Gökçentype boundary conditions being the most expensive. However, one could choose a simpler slip boundary condition that would not increase performance much and yet would give fairly accurate results. Therefore, CFD remains a viable option for the prediction of surface properties for a hypersonic vehicle in a moderately rarefied simple gas.

\section{Acknowledgments}

A. J. Lofthouse gratefully acknowledges the support of the U.S. Air Force Institute of Technology. This work is also sponsored in part by the Space Vehicle Technology Institute, under NASA grant NCC3-989 with joint sponsorship from the U.S. Department of Defense and the U.S. Air Force Office of Scientific Research through grant FA9550-05-1-0115. The generous use of NASA highperformance computing resources was indispensable to this investigation and is greatly appreciated.

\section{References}

[1] Bird, G. A., Gas Dynamics and the Direct Simulation of Gas Flows, Oxford University Press, Oxford, 1994.

[2] Bird, G. A., "Breakdown of Translational and Rotational Equilibrium in Gaseous Expansions," AIAA Journal, Vol. 8, No. 11, 1970, pp. 19982003.

[3] Tiwari, S., "Coupling of the Boltzmann and Euler Equations with Automatic Domain Decomposition," Journal of Computational Physics, Vol. 144, Aug. 1998, pp. 710-726. doi:10.1006/jcph.1998.6011

[4] Garcia, A. L., Bell, J. B., Crutchfield, W. Y., and Alder, B. J., "Adaptive Mesh and Algorithm Refinement Using Direct Simulation Monte Carlo," Journal of Computational Physics, Vol. 154, No. 1, 1999, pp. 134-155.

doi:10.1006/jcph.1999.6305

[5] Camberos, J. A., Schrock, C. R., McMullan, R. J., and Branam, R. D., "Development of Continuum Onset Criteria with Direct Simulation Monte-Carlo Using Boltzmann's H-Theorem: Review and Vision," 9th AIAA/ASME Joint Thermophysics and Heat Transfer Conference, San Francisco, CA, AIAA Paper 2006-2942, June 2006.

[6] Boyd, I. D., Chen, G., and Candler, G. V., "Predicting Failure of the Continuum Fluid Equations in Transitional Hypersonic Flows," Physics of Fluids, Vol. 7, No. 1, Jan. 1995, pp. 210-219. doi: $10.1063 / 1.868720$

[7] Wang, W.-L., "A Hybrid Particle/Continuum Approach for Nonequilibrium Hypersonic Flows," Ph.D. Thesis, Univ. of Michigan, Ann Arbor, MI, 2004.

[8] Lofthouse, A. J., Boyd, I. D., and Wright, M. J., "Effects of Continuum Breakdown on Hypersonic Aerothermodynamics," Physics of Fluids, Vol. 19, No. 2, 2007, p. 027105. doi:10.1063/1.2710289

[9] Dietrich, S., and Boyd, I. D., "Scalar and Parallel Optimized Implementation of the Direct Simulation Monte Carlo Method," Journal of Computational Physics, Vol. 126, No. 2, 1996, pp. 328-342. doi:10.1006/jcph.1996.0141

[10] Sun, Q., Cai, C., Boyd, I. D., Clemmons, J. H., and Hecht, J. H., "Computational Analysis of High- Altitude Ionization Gauge Flight 
Measurements," Journal of Spacecraft and Rockets, Vol. 43, No. 1, 2006, pp. 186-193.

[11] Scalabrin, L. C., and Boyd, I. D., "Development of an Unstructured Navier-Stokes Solver for Hypersonic Nonequilibrium Aerothermodynamics," 38th AIAA Thermophysics Conference, Toronto, Ontario Canada, AIAA Paper 2005-5203, June 2005.

[12] Scalabrin, L. C., and Boyd, I. D., "Numerical Simulation of Weakly Ionized Hypersonic Flow for Reentry Configurations," 9th AIAA/ ASME Joint Thermophysics and Heat Transfer Conference, San Fransisco, CA, AIAA Paper 2006-3773, June 2006.

[13] Schwartzentruber, T. E., Scalabrin, L. C., and Boyd, I. D., "Hybrid Particle-Continuum Simulations of Non-Equilibrium Hypersonic Blunt Body Flow Fields," 9th AIAA/ASME Joint Thermophysics and Heat Transfer Conference, San Francisco, CA, AIAA Paper 2006-3602, June 2006
[14] Lockerby, D. A., Reese, J. M., Emerson, D. R., and Barber, R. W., "Velocity Boundary Condition at Solid Walls in Rarefied Gas Calculations," Physical Review E (Statistical Physics, Plasmas, Fluids, and Related Interdisciplinary Topics), Vol. 70, 2004, Paper 017303. doi:10.1103/PhysRevE.70.017303

[15] Gökçen, T., and MacCormack, R. W., "Nonequilibrium Effects for Hypersonic Transitional Flows Using Continuum Approach," 27th Aerospace Sciences Meeting, Reno, NV, AIAA Paper 1989-0461, Jan. 1989.

[16] Gökçen, T., MacCormack, R. W., and Chapman, D. R., "Computational Fluid Dynamics Near the Continuum Limit," AIAA Paper 1987$1115,1987$.

[17] Lockerby, D. A., Reese, J. M., and Gallis, M. A., "Capturing the Knudsen Layer in Continuum-Fluid Models of Nonequilibrium Gas Flows," AIAA Journal, Vol. 43, No. 6, June 2005, pp. 1391-1393. 